\section{temporomandibulares en niños y su relación con la lactancia artificial con uso del biberón}

\section{Temporomandibular disorders in children and its relation to artificial lactation with bottle-feeding}

\section{Artículo Original}

Irene Espinosa-De Santillana 1,a, Lilian Ortega-Oviedo 1,b Gabriel Muñoz-Quintana ${ }^{1, c}$, Teresita Romero-Ogawa ${ }^{2, c}$, Indiana Torres-Escobar 2 ,a

${ }^{1}$ Benemérita Universidad Autónoma de Puebla, Facultad de Estomatología, Puebla, México.

2 Benemérita Universidad Autónoma de Puebla, Facultad de Medicina, Puebla, México.

a Doctorado en Ciencias

${ }^{\mathrm{b}}$ Maestría en Estomatología.

${ }^{c}$ Maestría en Ciencias.

\section{Correspondencia:}

Irene Espinosa De Santillana: irene.espinosa@correo. buap.mx

31 poniente 1304 Colonia Volcanes, CP 72104. Puebla, Puebla. México.

ORCID: 0000-0002-9055-2460

\section{Coautores:}

Lilian Ortega Oviedo: lilian.ortega@alumno.buap.mx ORCID: 0000-0002-1132-3919

Gabriel Muñoz Quintana: gabriel.munoz@correo.buap.mx ORCID: 0000-0001-7606-4359

Teresita Romero Ogawa: teresita.romero@correo.buap.mx ORCID: 0000-0002-7519-8029

Indiana Torres Escobar: indiana.torres@correo.buap.mx ORCID: 0000-0002-5013-2787

\section{Resumen}

Objetivo. Establecer la relación de los trastornos temporomandibulares (TTM) de niños de 8 a 10 años, con lactancia artificial exclusiva con uso del biberón. Métodos. Estudio de casos y controles, (pareados por edad y sexo). Se incluyeron 38 nińos, sin alteraciones del crecimiento ni parafunciones bucales severas, previo consentimiento (madres) y asentimiento (niños) informado. Los participantes fueron diagnosticados con TTM (19 casos) o sin diagnóstico de TTM (19 controles) utilizando los criterios diagnósticos para los TTM (CD/TTM), previa estandarización de la investigadora; kappa inter 0,98 e intraobservador 0,73 . Los antecedentes de lactancia fueron registrados con cuestionario $e x$ profeso: tipo y tiempo de administración y datos generales del niño y la madre. Se calculó estadística descriptiva e inferencial; Ji cuadrada para identificar la asociación entre TTM y lactancia artificial exclusiva con uso del biberón, y razón de posibilidades (OR), con valor significativo $\leq 0,05$. Resultados. Edad 8,95 $\pm 0,84$ años, $57,9 \%$ sexo masculino, sin diferencias por grupos en la edad y escolaridad de las madres $(\mathrm{p}>0,05)$. El tipo de TTM más común fue dolor muscular $(57,9 \%)$, seguido de luxación del disco con reducción y su combinación $(21,1 \%)$. La media de apertura bucal, sitios musculares y articulares doloridos fue estadísticamente diferente entre los casos y controles $(\mathrm{p}<0,05)$. El porcentaje de alimentación con lactancia artificial exclusiva con uso del biberón fue igual en ambos grupos $(57,9 \% \mathrm{p}=1,00)$, el OR calculado fue de 1 con IC95\% de 0,27-3,60. Conclusión. No se encontró relación entre la lactancia artificial con el uso del biberón y los TTM. El tipo de TTM más frecuente fue dolor muscular.

Palabras clave: Niños; Conducta en la lactancia; Asimetría facial; Trastornos de la articulación temporomandibular; Odontología pediátrica (fuente DeCS BIREME).

\footnotetext{
Abstract

Objective. To establish the relationship of temporomandibular disorders (TMD) in children aged 8 to 10 years, with exclusive artificial feeding. Methods. Case-control study (matched by age and sex). 38 children were included, without growth disturbances or severe oral parafunctions, with prior informed consent (mothers) and assent (children).
}

\section{Editora:}

Maria Eugenia Guerrero Acevedo

Universidad Nacional Mayor de San Marcos, Perú.

Conflicto de intereses: la Dra. Indiana Torres Escobar declara que ha recibido apoyo para investigación de Merck Sharp \& Dohme and Bristol. El resto de los autores declara no tener conflicto de intereses.

Fuente de financiamiento: no se recibió ningún financiamiento.

Recibido: 16/06/2020

Aceptado: 30/08/2020

Publicado: $16 / 11 / 2020$

(C) Los autores. Este artículo es publicado por la revista Odontología Sanmarquina de la Facultad de Odontología, Universidad Nacional Mayor de San Marcos. Este es un artículo de acceso abierto, distribuido bajo los términos de la licencia Creative Commons Atribucion - No Comercia_Compartir Igual 4.0 Internacional. (http://creativecommons.org/licenses/by-nc-sa/4.0/) que permite el uso no comercial, distribución y reproducción en cualquier medio, siempre que la obra original sea debidamente citada. 
Participants were diagnosed with TMD (19 cases) or without TMD diagnosis (19 controls) using the Diagnostic Criteria for TMD (CD / TMD), after standardization by the researcher; kappa inter 0.98 and intraobserver 0.73 . The history of breastfeeding was recorded with an express questionnaire: type and time of administration, and general data of the child and the mother. Descriptive and inferential statistics were calculated; Chi square analysis was performed to identify the association between TMD and exclusive artificial feeding, and odds ratio (OR), with a significant value of $\leq 0.05$. Results. Age $8.95 \pm 0.84$ years, $57.9 \%$ male, with no differences in age or education of the mother between groups ( $p>0,05$ ). The most common type of TMD was muscle pain $(57.9 \%)$, followed by disc luxation with reduction and combination (21.1\%). The mean mouth opening, muscle and joint pain sites were statistically different between cases and controls $(\mathrm{p}<0.05)$. The percentage of exclusive artificial feeding was the same in both groups $(57.9 \% \mathrm{p}=1.00)$, the calculated OR was 1 with a $95 \% \mathrm{CI}$ of $0.27-3.60$. Conclusions. Artificial feeding with the use of a bottle was not related to TMD. The most common type of TMD was muscle pain.

Keywords: Children; Sucking behaviors; Facial asymmetry; Temporomandibular disorders; Pediatric Dentistry (source: MeSH NLM).

\section{Introducción}

Los trastornos temporomandibulares (TTM), son un grupo de afecciones musculoesqueléticas y neuromusculares que incluyen varios signos y síntomas clínicos implicados en los músculos de la masticación, la articulación temporomandibular y las estructuras asociadas ${ }^{1}$. Actualmente estudios diversos reportan que los infantes y adolescentes presentan signos y síntomas de TTM ${ }^{2-4}$, específicamente las niñas ${ }^{5}$. Las prevalencias de TTM en los niños, varían considerablemente desde el 7,3\% hasta el 30,4\% ${ }^{6}$. La diversidad de instrumentos utilizados para su diagnóstico, ha contribuido a la heterogeneidad en las prevalencias 7. La etiología de los TTM en los niños es compleja y multifactorial. Factores múltiples han sido asociados en esta población, tales como: la mordida cruzada y las parafunciones bucales ${ }^{8}$. Los factores psicosociales han sido ampliamente estudiados y asociados a los TTM en la población infantil. Al-Khotani $e t$ al. ${ }^{9}$ en 2016 realizó un estudio transversal cuyo objetivo fue evaluar los problemas psicosociales en niños y adolescentes y su asociación con los TTM. Los resultados permitieron evidenciar que los niños con TTM dolorosos, presentan mayores frecuencias de ansiedad, depresión, quejas somáticas y conductas agresivas que los controles sanos $(\mathrm{p}<0,05)$. Adicionalmente, Kobayashi et al. ${ }^{10}$ en 2017 en un estudio transversal que evaluó los niveles de cortisol salival y los síntomas de ansiedad en niños y adolescentes brasileños con TTM $(\mathrm{n}=76)$ y controles sanos $(\mathrm{n}=38)$ demostraron que, si bien los niveles de cortisol salival no mostraron diferencias entre los grupos, la Escala de Ansiedad Multidimensional en su versión brasileña, si evidenció mayor frecuencia de ansiedad $(p=0,0007)$ en los niños y adolescentes con TTM. Estudios más recientes han fortalecido la evidencia que los aspectos psicosociales se asocian fuertemente con los TTM ${ }^{11,12}$. Por otro lado, también ha quedado demostrada la importancia del vínculo entre los padres y los hijos y su impacto sobre el estrés y la frecuencia de TTM en niños y adolescentes. Şermet Elbay et al. ${ }^{13}$ en 2017 demostraron que los niños viviendo en instituciones de asistencia a la niñez, presentan frecuencias mayores de TTM, parafunciones y estrés, que los niños viviendo con sus padres.

Adicionalmente, algunos factores como la lactancia artificial, pudieran comprometer el crecimiento y desarrollo del infante ${ }^{14-16}$. De acuerdo con Sánchez-Sánchez et al. la lactancia artificial, se asocia con el diagnóstico de los TTM, dada la alteración de la función de succión del bebé ${ }^{17}$, además de la ausencia del vínculo que representa la alimentación al seno materno. La lactancia artificial se establece como la sustitución de seno materno por el biberón, con fórmulas de leche adaptadas para la digestión correcta del bebé. En esta, la leche se obtiene sin esfuerzo y la duración de la succión se reduce notablemente ${ }^{18}$. Desafortunadamente, en muchas partes del mundo, incluido México, la lactancia materna ha seguido una tendencia decreciente ${ }^{19,20}$ mientras que poblaciones crecientes de lactantes son alimentadas con sustitutos de la leche materna; mediante el biberón. El lactante alimentado con biberón exclusivamente no realiza el mismo esfuerzo y, en consecuencia, disminuye su estimulación muscular correcta y pierde la sincronía de la respiración, lo que produce riesgo mayor de ser respirador bucal ${ }^{18}$. En virtud de que la lactancia artificial exclusiva con uso del biberón impacta las alteraciones en el crecimiento craneofacial y pudiera condicionar el desarrollo de los TTM, el objetivo de la presente investigación fue establecer la asociación de los TTM de niños de 8 a 10 años de edad, con el antecedente de haber recibido lactancia artificial exclusiva con uso del biberón.

\section{Métodos}

Bajo un diseño de estudio de casos y controles. Se evaluaron todos los niños de 8 a 10 años (72 infantes; $53,2 \%$ varones), de una escuela primaria pública previo consentimiento de las madres y asentimiento informado de los participantes de forma voluntaria. El protocolo de estudio, el consentimiento y asentimiento informado fueron aprobados por el Comité de Investigación de la Facultad de Estomatología de la Benemérita Universidad Autónoma de Puebla. 
De acuerdo con los criterios de selección, ningún niño presentó alteraciones clínicas del crecimiento craneofacial ni parafunciones bucales severas, ya fueran reportadas por ellos mismos o por los padres o con evidencia clínica de las anteriores. Posteriormente, del total de niños evaluados, se eliminaron 3 (2 niños y 1 niña) por no entender o seguir las instrucciones en la aplicación del instrumento. Para establecer o descartar el diagnóstico de TTM, se usaron los Criterios Diagnósticos para los trastornos temporomandibulares ${ }^{21}$ en su versión en español ${ }^{22}$. Los niños contestaron el cuestionario de antecedentes y fueron evaluados en un consultorio dental de la institución escolar por una investigadora previamente capacitada y estandarizada en la aplicación del instrumento (kappa interobservador de 0,98 e intraobservador de 0,73 ), con el estándar de referencia (IEDS); estandarizada en la universidad de Búfalo. El examen clínico propuesto por los CD/TTM, incluyó: la presencia y ubicación del dolor temporomandibular, el registro del patrón de la apertura bucal, de los recorridos (en milímetros) de las aperturas y lateralidades mandibulares, la presencia de ruidos articulares (chasquido o crepitación) en los movimientos mandibulares, la presencia de dolor al momento de realizar los movimientos, mialgias (20 sitios musculares) y artralgias (4 sitios articulares). De los 72 niños evaluados, se incluyeron 19 casos con algún diagnóstico confirmado de TTM; lo cual arrojó una prevalencia del 27,5\% y 19 controles que no presentaron ningún diagnóstico de TTM, pareados por edad y sexo con los casos. Para registrar los antecedentes de lactancia de los nińos, se diseñó un cuestionario ex profeso para la madre que incluyó: tipo de lactancia y tiempo de administración de la lactancia artificial con uso del biberón exclusivo, así como los datos generales del niño y de la madre. La lactancia artificial fue considerada cuando las madres expresaron haber proporcionado lactancia artificial exclusiva durante al menos los primeros 6 meses de vida del infante.

Análisis estadístico. Se calculó estadística descriptiva: medidas de tendencia central y dispersión, frecuencias y porcentajes de acuerdo con las escalas y las variables establecidas. Posteriormente se calculó estadística inferencial; prueba de asociación de Ji cuadrada entre el diagnóstico de trastornos temporomandibulares y la lactancia artificial, y razón de posibilidades (OR). Las pruebas estadísticas anteriores se interpretaron con un valor significativo $\leq 0,05$.

\section{Resultados}

Las características demográficas de la muestra estudiada por grupo (casos y controles) se presentan en la Tabla 1. La media de edad cercana a los 9 ańos y una participación superior de niños con respecto a las niñas $(57,9 \%)$. La igualdad en la edad media y el porcentaje de participación del sexo demuestra el efecto del pareo. La edad de las madres osciló entre los 26 y 51 años, con una media entre los 34 y los 38 años. La edad y la escolaridad de las madres de los casos, en relación con los controles, no denotó diferencias estadísticamente significativas; $\mathrm{p}=0,31$ y $\mathrm{p}=0,67$ respectivamente.

El diagnóstico de TTM y los signos y síntomas relacionados con los TTM se aprecian en la tabla 2. El tipo de TTM diagnosticado más frecuentemente en los niños fue el dolor muscular $(57,9 \%)$, aunque también se presentó luxación del disco con reducción en más del $20 \%$ de los niños, al igual que el diagnóstico combinado de los dos anteriores. Adicionalmente se reportaron algunos signos

Tabla 1. Descripción de la población por diagnóstico

\begin{tabular}{|c|c|c|c|c|c|c|}
\hline & & \multicolumn{2}{|c|}{$\begin{array}{c}\begin{array}{c}\text { Niños con TTM } \\
n=19\end{array} \\
\end{array}$} & \multicolumn{2}{|c|}{$\begin{array}{c}\text { Niños sin TTM } \\
n=19\end{array}$} & \multirow[b]{2}{*}{$\mathrm{p}$} \\
\hline \multirow{7}{*}{ Edad del niño } & & $\mathbf{n}$ & $\%$ & $\mathbf{n}$ & $\%$ & \\
\hline & 8 años & 7 & 36,8 & 7 & 36,8 & \multirow{3}{*}{$* 1,00$} \\
\hline & 9 años & 6 & 31,6 & 6 & 31,6 & \\
\hline & \multirow[t]{4}{*}{10 años } & 6 & 31,6 & 6 & 31,6 & \\
\hline & & Media & DE & Media & DE & \multirow{3}{*}{$* * 1,00$} \\
\hline & & 8.9 & 0,8 & 8,9 & 0,8 & \\
\hline & & $\mathbf{n}$ & $\%$ & $\mathbf{n}$ & $\%$ & \\
\hline \multirow[t]{2}{*}{ Sexo } & Femenino & 8 & 42,1 & 8 & 42,1 & \multirow{2}{*}{${ }^{*} 1,00$} \\
\hline & Masculino & 11 & 57,9 & 11 & 57,9 & \\
\hline \multirow{3}{*}{ Edad de la madre } & & Media & $\mathrm{DE}$ & Media & $\mathrm{DE}$ & \multirow{3}{*}{$* * 0,31$} \\
\hline & & 33,9 & 7,7 & 37,6 & 6,4 & \\
\hline & & $\mathbf{n}$ & $\%$ & $\mathbf{n}$ & $\%$ & \\
\hline \multirow{2}{*}{ Nivel de escolaridad de la madre } & Básico & 16 & 84,2 & 15 & 78,9 & \multirow{2}{*}{$* 0,67$} \\
\hline & $\mathrm{MS} / \mathrm{S}$ & 3 & 15,8 & 4 & 21,1 & \\
\hline
\end{tabular}

MS/S Medio Superior/Superior

*Ji cuadrada

$* *$ t de Student 
y síntomas relacionados con el diagnóstico de TTM: la media de apertura mandibular máxima sin dolor se notó disminuida en el grupo de los casos (34,4 milímetros) vs los controles (39,9 milímetros). Adicionalmente, la media de músculos $(8,3$ vs 1,4$)$ y sitios articulares $(1,3$ vs $0,4)$ doloridos fue significativamente mayor en el grupo de los casos, con relación a los controles. Destaca que los niños sin TTM, también presentaron porcentajes considerables de patrón de apertura mandibular alterado y ruidos articulares, específicamente chasquido.

Finalmente, la Tabla 3 permite la comparación de la lactancia artificial exclusiva con uso del biberón entre los casos y los controles y denota que ésta tuvo el mismo porcentaje $(57,9 \%)$ entre los casos y los controles. Los resultados de la razón de posibilidades $(\mathrm{OR}=1$ con IC95\% 0,27-3,60) corroboran la falta de asociación. El tiempo en semanas que los niños recibieron alimentación artificial exclusiva con el uso del biberón, superó las 24 semanas $(27,3 \%$ vs $45,5 \%)$ y hasta las 52 semanas $(36,3 \%$ vs $27,3 \%)$, tanto en el grupo con TTM como en el grupo libre del diagnóstico respectivamente.

\section{Discusión}

En el presente estudio de investigación no se encontró una relación entre la lactancia artificial exclusiva y los TTM. Estos resultados, difieren de lo reportado por

Tabla 2. Características relacionadas con la evaluación clínica de TTM

\begin{tabular}{|c|c|c|c|c|c|c|c|}
\hline & \multicolumn{3}{|c|}{ Niños con TTM } & \multicolumn{3}{|c|}{ Niños sin TTM } & $\mathrm{p}$ \\
\hline Dx. TTM & \multicolumn{3}{|c|}{$\mathrm{n}=19$} & \multicolumn{3}{|c|}{$\mathrm{n}=19$} & - \\
\hline \multirow{5}{*}{ Tipo de TTM } & & n & $\%$ & & & & \\
\hline & Mialgia & 11 & 57,9 & & & & \\
\hline & $\begin{array}{l}\text { Desplazamiento del Disco } \\
\text { con Reducción (DDCR) }\end{array}$ & 4 & 21,1 & & & & - \\
\hline & Mialgia y DDCR & 4 & 21,1 & & & & \\
\hline & & $\mathbf{n}$ & $\%$ & & $\mathbf{n}$ & $\%$ & \\
\hline \multirow{3}{*}{ Patrón de apertura } & Recto & 15 & 78,9 & Recto & 16 & 84,2 & \multirow{2}{*}{$* 0,67$} \\
\hline & Alterado & 4 & 21,1 & Alterado & 3 & 15,8 & \\
\hline & Media & \multicolumn{2}{|c|}{ DE } & Media & \multicolumn{2}{|c|}{ DE } & \\
\hline \multirow[t]{2}{*}{ Apertura Confortable } & 34,4 & \multicolumn{2}{|c|}{7,3} & 39,89 & \multicolumn{2}{|c|}{4,7} & $* *<0,001$ \\
\hline & Media & \multicolumn{2}{|c|}{ DE } & Media & \multicolumn{2}{|c|}{ DE } & - \\
\hline \multirow[t]{2}{*}{ Apertura máxima } & 42,7 & \multicolumn{2}{|c|}{5,4} & 43,4 & \multicolumn{2}{|c|}{5,2} & $* * 0,67$ \\
\hline & $\mathbf{n}$ & \multicolumn{2}{|c|}{$\%$} & $\mathbf{n}$ & \multicolumn{2}{|c|}{$\%$} & - \\
\hline \multirow[t]{2}{*}{ Ruidos articulares } & 3 & \multicolumn{2}{|c|}{7,8} & 0 & \multicolumn{2}{|c|}{0} & ${ }^{*} 0,34$ \\
\hline & Media & \multicolumn{2}{|c|}{ DE } & Media & \multicolumn{2}{|c|}{ DE } & \\
\hline $\begin{array}{l}\text { Media de sitios musculares } \\
\text { doloridos a la palpación }\end{array}$ & 8,3 & \multicolumn{2}{|c|}{3,6} & 1,4 & \multicolumn{2}{|c|}{2} & $* *<0,001$ \\
\hline $\begin{array}{l}\text { Media de sitios articulares } \\
\text { doloridos a la palpación }\end{array}$ & 1,3 & \multicolumn{2}{|c|}{1,3} & 0,4 & \multicolumn{2}{|c|}{0,9} & ${ }^{* *} 0,02$ \\
\hline
\end{tabular}

*Ji Cuadrada

**t de Student

Tabla 3. Lactancia artificial exclusiva con biberón de acuerdo con el diagnóstico de TTM

\begin{tabular}{|c|c|c|c|c|c|c|}
\hline \multirow{3}{*}{$\begin{array}{l}\text { Administración de lactancia artifi- } \\
\text { cial exclusiva con biberón }\end{array}$} & \multirow{3}{*}{$\begin{array}{l}\text { Sí } \\
\text { No }\end{array}$} & \multicolumn{2}{|c|}{$\begin{array}{c}\text { Niños con TTM } \\
n=19\end{array}$} & \multicolumn{2}{|c|}{$\begin{array}{c}\text { Niños sin TTM } \\
n=19\end{array}$} & \multirow{3}{*}{$\frac{{ }^{*} \mathrm{p}}{1,00}$} \\
\hline & & 11 & 57,9 & 11 & 57,9 & \\
\hline & & 8 & 42,1 & 8 & 42,1 & \\
\hline & & \multicolumn{2}{|c|}{$\begin{array}{l}\text { OR } \\
1,00\end{array}$} & \multicolumn{2}{|c|}{$\begin{array}{c}\text { IC95\% } \\
0,27-3,60\end{array}$} & \\
\hline & & $\mathbf{n}$ & $\%$ & $\mathrm{n}$ & $\%$ & \\
\hline \multirow{4}{*}{$\begin{array}{l}\text { Tiempo de administración de } \\
\text { la lactancia artificial (medido en } \\
\text { semanas) }\end{array}$} & $0-12$ & 3 & 27,3 & 1 & 9,1 & \multirow{4}{*}{0,74} \\
\hline & 13 a 24 & 1 & 9,1 & 2 & 18,1 & \\
\hline & 25 a 52 & 3 & 27,3 & 5 & 45,5 & \\
\hline & Más de 52 & 4 & 36,3 & 3 & 27,3 & \\
\hline
\end{tabular}

\section{*Ji Cuadrada}


Sánchez-Sánchez et al. ${ }^{17}$ en el año 2017, en la ciudad de Madrid, España; quienes concluyeron que la lactancia artificial se relaciona con los TTM graves. La discrepancia en los resultados podría deberse a distintos factores; por un lado, a que la evaluación clínica para el diagnóstico de los TTM que ellos realizaron; fue con el uso de los CDI/TTM, los cuales ya han demostrado validez en sujetos desde los 12 ańos ${ }^{23}$. Sin embargo, en la metodología no se enuncia si hubo estandarización para el uso de dicho instrumento. A diferencia del estudio de Sánchez-Sánchez et al., en la presente investigación, el diagnóstico de TTM de los niños, se realizó con el uso de los Criterios Diagnósticos para los TTM, versión actualizada de los CDI/TTM. Los CD/TTM han demostrado una validez y confiabilidad superior a los CDI/ TTM en adultos ${ }^{21}$.

Adicionalmente, en el estudio de Sánchez-Sánchez et al. establecieron un diagnóstico de TTM grave; cuando el paciente presentó 6 signos o síntomas de TTM. En la presente investigación, ninguno de los niños presentó más de 6 signos o síntomas que permitieran diagnosticarlo como grave, de acuerdo con la clasificación empleada en el estudio de Sánchez-Sánchez et al. lo cual podría explicar la falta de asociación entre la lactancia artificial y el uso del biberón y los TTM en la investigación presente. Por otro lado, los nińos incluidos en el estudio anteriormente mencionado fueron mayores (hasta los 13 años), en comparación con la edad de los niños en la presente investigación, con solo 8 a 10 años. Así mismo, otro factor que pudiera marcar la diferencia en los resultados, es la tendencia a que conforme aumenta la edad en los niños, es mayor el riesgo de padecer TTM y mayor el grado de severidad. Por otro lado, la evaluación de la muestra en nuestra investigación fue pareada por edad y sexo, con un ligero predominio del sexo masculino, mientras que Sánchez-Sánchez et al, incluyeron una muestra de $50 \%$ de cada sexo y no se especificaba pareo entre los casos y los controles.

El diagnóstico de TTM más frecuentemente encontrado en la presente investigación fue el dolor muscular; lo anterior, coincide con el estudio de investigación descrito por Grosfeld et al. ${ }^{23}$ quienes concluyen que las prevalencias de diagnóstico de TTM en los niños son frecuentemente del tipo muscular. Sin embargo, Gatzi et $a l^{24}$ difieren en sus resultados; al encontrar predominio en el diagnóstico de ruidos articulares en su estudio de niños israelitas.

Uno de los hallazgos de la presente investigación fue que los niños del grupo control (que no cumplieron con los criterios de los CD/TTM para recibir el diagnóstico), presentaron signos y síntomas relacionados con los TTM. Los signos y síntomas encontrados en el grupo de niños sin TTM fueron: los patrones de apertura mandibular alterados y la presencia de ruidos articulares, específicamente chasquidos. Lo anterior, coincide con otros estudios relacionados que han encontrado patrones de apertura mandibular alterados y ruidos articulares en niños sin diagnóstico de TTM ${ }^{25,26}$.
Una de las debilidades del presente estudio es el uso de los CD/TTM, instrumento que, al momento de realizar el estudio, no contaba con validez y confiabilidad demostrada. Estudios recientes, posteriores a la investigación presente, han demostrado validez y confiabilidad de los CD/TTM, aún en niños desde los 7 años, aunque se proponen algunas modificaciones en el instrumento que no fueron aplicadas en esta investigación ${ }^{27}$.

Al evaluar la prevalencia de la lactancia artificial exclusiva con uso del biberón, se encontró que fue cercana al 60\% del total de la población evaluada, independientemente de pertenecer al grupo de casos o de controles. Estos porcentajes, a pesar de ser menores que los que describe la Encuesta Nacional de Salud en México ${ }^{19}$ y UNICEF ${ }^{20}$ que reportan prevalencias que van del $81,5 \%$ al $85,5 \%$, en zonas rurales y urbanas, respectivamente; demuestran que, la administración de la lactancia artificial exclusiva sigue en aumento en los últimos años. Otro factor importante es que dicha lactancia artificial se siguió administrando con uso del biberón aún después de los 6 meses de vida y hasta más de un año, con incorporación de otro tipo de dieta en los participantes; tanto en el grupo de infantes con TTM (63,6\%), como en el grupo sin el diagnóstico de TTM $(72,8 \%)$. Una debilidad del presente estudio fue la falta de registro de la dieta complementaria, posterior a los primeros 6 meses de vida en los cuales se administró lactancia artificial exclusiva con uso del biberón. Lo anterior, podría ser un factor de confusión para la interpretación de los resultados, debido a la importancia vital que representa lo anterior para el desarrollo adecuado del sistema estomatognático.

Los resultados de la presente investigación confirman las prevalencias considerables tanto de los TTM (27,5\%), como de la lactancia artificial exclusiva con uso del biberón (57,9\%).

Adicional al aspecto físico, es importante resaltar el impacto psicosocial que implica la sustitución de lactancia materna en los niños, específicamente en los primeros meses de vida ${ }^{24}$, lo cual pudiera impactar en los aspectos psicosociales ampliamente documentados como factores de riesgo para el desarrollo de los TTM ${ }^{9-12}$.

En la presente investigación, no se demostró relación entre la lactancia artificial y los TTM, probablemente debido a la etiología multifactorial que impacta el desarrollo de los trastornos y no solo en respuesta a factores individuales como en este caso, la lactancia artificial exclusiva con el uso del biberón. Sin embargo, es importante enfatizar a las madres sobre la importancia que tiene la lactancia materna, puesto que además de proporcionarle al recién nacido todo lo necesario para el crecimiento y desarrollo físico, social y emocional óptimo, produce un beneficio psicológico en el vínculo madre e hijo ${ }^{28}$. Por otro lado, si la lactancia materna no es posible, y se sustituye por la lactancia artificial exclusiva con el uso del biberón, su administración no debe superar los 6 meses de vida. 
En conclusión, el presente estudio concluye que no existe relación entre los TTM y la lactancia artificial exclusiva. Los niños que recibieron lactancia artificial con uso del biberón presentan la misma posibilidad de desarrollar trastornos temporomandibulares que aquellos alimentados al seno materno. Algunos signos y síntomas de TTM también están presentes en los niños que no presentan el diagnóstico, de acuerdo con el instrumento utilizado.

\section{Referencias bibliográficas}

1. de Leeuw R, Klasser G. The american academy of orofacial pain. Orofacial pain: Guidelines for assessment, diagnosis and management. [Internet]. Sixth edit. De Leeuw R, Klasser G, editors. Hanover Park, IL, USA: Quintessence Publishing Co, Inc.; 2018. 336 p. Disponible en: https://aaop.org/

2. Castelo PM, Gavião MBD, Pereira LJ, Bonjardim LR. Relationship between oral parafunctional/nutritive sucking habits and temporomandibular joint dysfunction in primary dentition. Int J Paediatr Dent [Internet]. 2005 Jan 1 [citado el 11 de junio de 2020];15(1):29-36. Disponible en: http://doi.wiley.com/10.1111/j.1365263X.2005.00608.x

3. Arenas Carreño M, Bloise Triana A, Carvajal Pabón M, Forero Santamaría C, Rodríguez Ciódaro A, Herrera Vivas M. Signos y síntomas de trastornos temporomandibulares en niños entre los 6 y los 13 años de edad. Serie de 50 casos. Univ Odontológica [Internet]. 2013 [citado el 11 de junio de 2020];32(69):1618. Disponible en: https://revistas.javeriana.edu.co/ index.php/revUnivOdontologica/article/view/SICI\%3A 2027-3444\%28201307\%2932\%3A69\%3C161\%3ASSTTEN\%3E2.0.CO\%3B2-6

4. Farsi NMA. Symptoms and signs of temporomandibular disorders and oral parafunctions among Saudi children. J Oral Rehabil [Internet]. 2003 Nov 26 [citado el 11 de junio de 2020];30(12):1200-8. Disponible en: http:// doi.wiley.com/10.1111/j.1365-2842.2003.01187.x

5. Minghelli B, Cardoso I, Porfírio M, Gonçalves R, Cascalheiro S, Barreto V, et al. Prevalence of temporomandibular disorder in children and adolescents from public schools in southern portugal. N Am J Med Sci [Internet]. 2014 [citado el 11 de junio de 2020];6(3):12632. Disponible en: https://pubmed.ncbi.nlm.nih. gov/24741551

6. Christidis N, Lindström Ndanshau E, Sandberg A, Tsilingaridis $\mathrm{G}$. Prevalence and treatment strategies regarding temporomandibular disorders in children and adolescents-A systematic review. J Oral Rehabil [Internet]. 2019 [citado el 11 de junio de 2020];46(3):291-301. Disponible en: http://doi.wiley.com/10.1111/joor.12759

7. Ramírez-Caro SN, Espinosa de Santillana IA, Muñoz-Quintana G. Prevalencia de trastornos temporomandibulares en niños mexicanos con dentición mixta. Rev Salud Publica [Internet]. 2015 [citado el 11 de junio de 2020];17(2):289-99. Disponible en: https:// revistas.unal.edu.co/index.php/revsaludpublica/article/ view/27958/62032

8. Perrotta S, Bucci R, Simeon V, Martina S, Michelotti A, Valletta R. Prevalence of malocclusion, oral parafunctions and temporomandibular disorder-pain in
Italian schoolchildren: An epidemiological study. J Oral Rehabil [Internet]. 2019 [citado el 11 de junio de 2020];46(7):joor.12794. Disponible en: https://onlinelibrary.wiley.com/doi/abs/10.1111/joor.12794

9. Al-Khotani A, Naimi-Akbar A, Gjelset M, Albadawi E, Bello L, Hedenberg-Magnusson B, et al. The associations between psychosocial aspects and TMD-pain related aspects in children and adolescents. J Headache Pain [Internet]. 2016 [citado el 11 de junio de 2020];17(1). Disponible en: https://www.proxydgb.buap.mx:2057/ login.aspx?direct=true $\& d b=a s n \& A N=114246016 \& l a n-$ $\mathrm{g}=\mathrm{es} \&$ site $=$ ehost-live

10. Kobayashi FY, Gavião MBD, Marquezin MCS, Fonseca FLA, Montes ABM, Barbosa T de S, et al. Salivary stress biomarkers and anxiety symptoms in children with and without temporomandibular disorders. Braz Oral Res [Internet]. 2017 [citado el 11 de junio de 2020];31:e78. Disponible en: https://www.proxydgb.buap.mx:2057/ login.aspx?direct=true\&db=mdc\&AN=29019550\&lan$\mathrm{g}=\mathrm{es} \&$ site $=$ ehost-live

11. Impellizzeri A, Di Benedetto S, De Stefano A, Monaco Guercio E, Barbato E, Galluccio G. General health \& psychological distress in children with temporomandibular disorder. Clin Ter [Internet]. 2019 [citado el 11 de junio de 2020];5:e321-7. Disponible en: http://www. proxydgb.buap.mx:2048/login?url=https $\% 3 \mathrm{~A} \% 2 \mathrm{~F} \%$ 2Fsearch.ebscohost.com\%2Flogin.aspx\%3Fdirect\%3Dtrue $\% 26 \mathrm{db} \% 3 \mathrm{Dmdc} \% 26 \mathrm{AN} \% 3 \mathrm{D} 31612186 \% 26$ lang\%3Des\%26site\%3Dehost-live

12. Marpaung C, van Selms MKA, Lobbezoo F. Prevalence and risk indicators of pain-related temporomandibular disorders among Indonesian children and adolescents. Community Dent Oral Epidemiol [Internet]. 2018 [citado el 11 de junio de 2020];46(4):400-6. Disponible en: http://doi.wiley.com/10.1111/cdoe.12382

13. Șermet Elbay Ü, Demirturk Kocasarac H, Elbay M, Kaya C, Uğurluel C, Baydemir C. Temporomandibular disorders and oral parafunction in children living with their parents and children living in institutional protective care: a comparative study. Int Dent J [Internet]. 2017 [citado el 11 de junio de 2020];1:20-8. Disponible en: http://www.proxydgb.buap.mx:2048/login?url=https\%3A\%2F\%2Fsearch.ebscohost.com\%2Flogin. aspx $\% 3$ Fdirect $\% 3$ Dtrue $\% 26 \mathrm{db} \% 3 \mathrm{Dc} 8 \mathrm{~h} \% 26 \mathrm{AN} \%$ 3D121248650\%26lang\%3Des\%26site\%3Dehost-live

14. López Rodríguez YN. Función motora oral del lactante como estímulo de crecimiento craneofacial / Infant Oral Motor Function as a Stimulus for Craniofacial Growth. Univ Odontol. 2016 Jun 28;35(74):127-39.

15. Morales-Chávez MC S-DVR. Influencia de la lactancia materna en la aparición de hábitos parafuncionales y maloclusiones: estudio transversal. Univ Odontológicaodontologica [Internet]. 2014 [citado el 11 de junio de 2020] Disponible en: http://dx.doi.org/10.11144/Javeriana.\%0Auo33-71.ilma

16. Carrascoza KC, Possobon RDF, Tomita LM, De Moraes ABA. Consequences of Bottle-Feeding to the Oral Facial Development of Initially Breastfed Children. J Pediatr (Rio J). 2006 [citado el 11 de junio de 2020];82(5):395-7.

17. Sánchez-Sánchez ME, Gallardo-López NE, San Román-Calvar P, Vázquez-Palacios MI. Factores etiológicos 
de la disfunción craneomandibular en una población de niños españoles. Rev Odontol Pediátrica. 2018 [citado el 11 de junio de 2020];13;16(2).

18. Ustrell JM, Sánchez-Molins M. Fisiología bucal infantil: función y crecimiento de la cavidad oral del lactante [Internet]. Vol. 4, Matronas Profesión. 2003 [citado el 11 de junio de 2020]. Disponible en: http://orthodonticworld. com/default 1. htm

19. Encuesta Nacional de Salud y Nutrición 2012 [Internet]. [citado el 11 de junio de 2020] México; Disponible en: https://ensanut.insp.mx/encuestas/ensanut2012/doctos/ informes/ENSANUT2012ResultadosNacionales.pdf

20. UNICEF México [Internet]. [citado el 11 de junio de 2020] Disponible en: https://www.unicef.org/mexico/

21. Schiffman E, Ohrbach R, Truelove E, Look J, Anderson G, Goulet J-P, et al. Diagnostic Criteria for Temporomandibular Disorders (DC/TMD) for Clinical and Research Applications: Recommendations of the International RDC/TMD Consortium Network* and Orofacial Pain Special Interest Group $\dagger$. J Oral Facial Pain Headache [Internet]. 2014 [citado el 11 de junio de 2020]; 28(1):6-27. Disponible en: https://pubmed.ncbi.nlm. nih.gov/24482784

22. Ohrbach R, Gonzalez Y, Castrillón E, Oyarzo JF, Espinoza de Santillana I, Ortiz F, et al. Diagnostic Criteria for Temporomandibular Disorders: Assessment Instruments [Internet]. International RDC/TMD Consortium Network. 2016 [citado el 11 de junio de 2020] Disponible en: https://buffalo.app.box.com/s/u9jd6bzk7sfai7o6wxwvsjchgtyu4zvh
23. Olga G, Czarnecka B. Musculo-articular disorders of the stomatoganthic system in school children examined according to clinical criteria. J Oral Rehabil [Internet]. 1977 [citado el 11 de junio de 2020];4(2):193-200. Disponible en: http://doi.wiley.com/10.1111/j.1365-2842.1977. tb00983.x

24. Gazit E, Lieberman M, Eini R, Hirsch N, Serfaty V, Fuchs C, et al. Prevalence of mandibular dysfunction in 10-18 year old Israeli schoolchildren. J Oral Rehabil [Internet]. [citado el 11 de junio de 2020];11(4):307-17. Disponible en: https://doi.org/10.1111/j.1365-2842.1984. tb00581.x

25. Silvia R, Espinosa I, Gabriel M. Descripcion del patron y recorrido de la apertura mandibular en ninos con denticion mixta. Rev Odontopediatria Latinoam. 2011 Nov $1 ; 1: 195-7$.

26. Cortese S, Biondi A, Fridman D, Guitelman I, Farah C. Assessment of Mandibular Movements in 10 to 15 Yearold Patients With and Without Temporomandibular Disorders. Acta Odontol Latinoam. 2015;28:237-43.

27. Restrepo C, Suarez N, Moratto N, Manrique R. Content and construct validity of the Diagnostic Criteria for temporomandibular Disorders Axis I for children. J Oral Rehabil [Internet]. 2020 [citado el 11 de junio de 2020];joor.12957. Disponible en: https://onlinelibrary. wiley.com/doi/abs/10.1111/joor.12957

28. Pilonieta Ortiz G, Torres Murillo EA. Implicaciones de la lactancia materna en odontopediatría. MedUNAB. 2003;6(17):89-92. 
